\title{
Histoire du Collège Sainte-Marie-de-Monnoir (1853-1912)
}

\section{Jean-Jacques Robillard}

Volume 47, 1980

URI : https://id.erudit.org/iderudit/1007106ar

DOI : https://doi.org/10.7202/1007106ar

Aller au sommaire du numéro

Éditeur(s)

Les Éditions Historia Ecclesiæ Catholicæ Canadensis Inc.

ISSN

0318-6172 (imprimé)

1927-7067 (numérique)

Découvrir la revue

Citer cet article

Robillard, J.-J. (1980). Histoire du Collège Sainte-Marie-de-Monnoir (1853-1912). Sessions d'étude - Société canadienne d'histoire de l'Église catholique, 47, 35-53. https://doi.org/10.7202/1007106ar

Tous droits réservés @ Les Éditions Historia Ecclesiæ Catholicæ Canadensis Inc., 1980
Ce document est protégé par la loi sur le droit d'auteur. L'utilisation des services d’Érudit (y compris la reproduction) est assujettie à sa politique d'utilisation que vous pouvez consulter en ligne.

https://apropos.erudit.org/fr/usagers/politique-dutilisation/ 


\section{Histoire du Collège Sainte-Marie-de-Monnoir $(\mathbf{1 8 5 3 - 1 9 1 2})^{1}$}

L'histoire du Collège Sainte-Marie-de-Monnoir commence avec la nomination de $\mathrm{M}^{\mathrm{gr}}$ Jean-Charles Prince au nouveau siège épiscopal de Saint-Hyacinthe érigé le 8 juin $1852^{2}{ }^{2}$. À cette époque, M. Édouard Crevier était curé de Saint-Hyacinthe depuis 1832. Le nouvel évêque, ne voulant pas être à la charge de ses diocésains, avait formé le projet de devenir lui-même curé de l'unique paroisse de la nouvelle ville épiscopale tout en se faisant remplacer par un prêtre pour prendre soin de la cure. Or, comme il n'était pas question pour l'évêque d'enlever à l'abbé Crevier son titre de curé pour en faire uniquement un vicaire actuel qui exercerait le ministère à sa place, il résolut donc de lui confier une nouvelle paroisse. Ainsi décida-t-il de le nommer à la paroisse Sainte-Marie-de-Monnoir ${ }^{3}$.

En assignant $\mathrm{M}$. Crevier à Sainte-Marie-de-Monnoir, $\mathrm{M}^{\mathrm{gr}}$ Prince lui demanda de mener à bien un projet qui avait été amorcé par l'abbé Liboire-Henri Girouard, son prédécesseur, mais qui traînait en longueur ${ }^{4}$. En effet, quelques années auparavant, M. Girouard

1 Il s'agit d'un résumé des deux premiers chapitres de ma thèse de maîtrise, laquelle fut présentée à l'Université d'Ottawa à l'automne de 1979. Voici la liste des sigles utilisés dans les notes :

$\begin{array}{ll}\text { AESH } & \text { Archives de l'Évêché de Saint-Hyacinthe } \\ \text { APC } & \text { Archives publiques du Canada (Ottawa) } \\ \text { APM } & \text { Archives du Presbytère de Marieville } \\ \text { APSQ } & \text { Archives du Petit Séminaire de Québec } \\ \text { ASCH } & \text { Archives des Sœurs de la Charité de Saint-Hyacinthe } \\ \text { ASSH } & \text { Archives du Séminaire de Saint-Hyacinthe } \\ \text { DP-57M } & \text { Documents paroissiaux : Dossier 57-Marieville } \\ \text { RL } & \text { Registre des lettres des évêques de Saint-Hyacinthe } \\ \text { UL } & \text { Université Laval } \\ \text { FSSM } & \text { Fonds Sainte-Marie-de-Monnoir }\end{array}$

2 Voir Mandements, lettres pastorales et circulaires des Évêques de Saint-Hyacinthe, t. 1, Montréal, C.-O. Beauchemin et Fils, 1888, p. 9-10.

3 AESH, RL, Série I, v. 1, Mgr J.-C. Prince à l'abbé Ed. Crevier, curé de Saint-Hyacinthe (25 octobre 1852).

4 Voir AESH, DP-57M, M. Ed. Crevier à Mgr J.-C. Prince (16 novembre 1852). 
avait fait l'acquisition de la maison d'un certain docteur Davignon en vue d'établir une maison d'éducation. Après avoir parlé de son projet au nouveau curé, l'évêque écrivit au juge Rolland, seigneur de Monnoir, pour lui demander de lui faire connaître la « remise » qu'il lui ferait si jamais il acquérait la propriété de M. Girouard pour ouvrir une maison d'éducation à Sainte-Marie-de-Monnoir ${ }^{5}$. Le juge Rolland, se disant heureux de contribuer ainsi à la mise sur pied d'un établissement d'éducation dans sa seigneurie, lui répondit qu'il n'exigerait aucune indemnité ${ }^{6}$.

Devant une réponse aussi favorable, $\mathrm{M}^{*}$ Prince incita l'abbé Crevier à présenter le projet à l'assemblée des marguilliers pour savoir si la Fabrique n'accepterait pas d'acheter cette maison dans le but d'établir une maison d'enseignement, s'engageant de son côté à procurer des frères ou des sœurs dès l'automne de $1853^{7}$.

M. Crevier présenta le projet de l'évêque à l'assemblée des paroissiens du 25 décembre 1852. Les paroissiens favorisèrent l'éta-

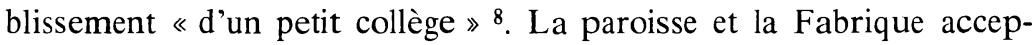
taient de se porter acquéreurs de la maison et du terrain pour $£ 350$. M. Crevier, enthousiasmé par le projet, fit part à $\mathrm{M}^{\mathrm{gr}}$ Prince de ses vues sur ce collège qui n'était encore qu'à l'état de projet.

Voici comme la chose pourra s'arranger. La paroisse et la fabrique payeront les $£ 350$. pour le terrain et les dépenses et le curé se chargera des agrandissements et des améliorations aux bâtiments. J'allongerai la maison actuelle d'à peu près autant et je mettrai le tout à deux étages. Il n'y a pas à faire autrement si on ne veut pas gaspiller son argent et ne rien faire de bon et de suffisant pour nos vues. Nous recevrons des frères pour le français et l'anglais et - à un autre temps les détails. Je vais ouvrir une souscription dans la paroisse. Nous pétitionnerons pour avoir un acte d'incorporation, du Curé, $1^{\text {er }}$ Marguillier et du $1^{\text {er }}$ commissaire d'école. Nous aurons des argents du gouvernement par la suite. Notre représentant est favorable à la chose 9 .

5 Voir AESH, RL, Série I, v. 1, Mgr J.-C. Prince au juge Rolland, Seigneur de Monnoir (26 novembre 1852).

6 AESH, DP-57M M. le juge Rolland Seigneur de Monnoir, à Mgr J.-C. Prince (2 décembre 1852).

7 Voir AESH, RL, Série I, v. 1, Mgr J.-C. Prince à M. Ed. Crevier (20 décembre 1852).

8 AESH, DP-57M, M. Ed. Crevier à Mgr J.-C. Prince (30 décembre 1852).

9 Ibid. 
Mais peu de temps après, M. Crevier se vit offrir par les paroissiens la maison d'école du village en vue de l'établissement d'un couvent pour les jeunes filles ${ }^{10}$. Devant cette offre, M. Crevier se ravisa et décida qu'il installerait le couvent pour les jeunes filles dans la maison du docteur Davignon car elle lui paraissait mieux convenir à l'établissement d'une communauté religieuse de femmes, puis il agrandirait la maison d'école pour y établir le collège pour les garçons ${ }^{11}$.

M. Crevier fit en sorte que les travaux avancent rondement. Cinq mois après avoir obtenu la permission de l'évêque, il pouvait lui écrire : «la maçonnerie est finie, le comble et la couverture sont posés, les châssis sont à se poser, etc. En un mot la maison sera logeable au $1^{\text {er }}$ octobre ${ }^{12}$. Et en effet, à l'automne de 1853, les paroissiens avaient la joie d'assister à l'ouverture officielle de deux maisons d'éducation secondaire dans leur localité : le Collège SainteMarie-de-Monnoir pour les garçons, et le Couvent des Sœurs de la Présentation de Marie pour les filles.

Cependant, il ne faudrait pas croire que cela se fit sans heurt. Dans sa lettre à $\mathrm{M}^{\mathrm{gr}}$ Prince, datée du 30 décembre 1852, M. Crevier avait commencé à parler des matières qui seraient enseignées au Collège. En fait, l'évêque et $M$. Crevier ne s'entendaient pas sur la nature et le genre de maison d'éducation que devait être le Collège. Pour M. Crevier, le petit collège qu'il veut établir à Sainte-Mariede-Monnoir doit être un véritable Collège. C'est ainsi que le 7 août 1853, M. Crevier faisait parvenir à $\mathrm{M}^{\mathrm{*} r}$ Prince un plan d'études pour un cours complet de huit années. Ce plan d'études, l'évêque ne l'accepte pas; pour lui, il ne faut pas «songer au latin ni au grec pour l'Académie de Sainte-Marie-de-Monnoir», car les «Évêques, dans une Congrégation privée, lors du Concile Provincial » de 1851, ont décidé "que l'on ne permettra plus l'ouverture de nouveaux collèges d'ici à bien des années ${ }^{13}$.

Pour $\mathrm{M}^{\mathrm{rr}}$ Prince, la nouvelle maison d'éducation de M. Crevier devait servir de "collège ou d'académie » ${ }^{14}$, et non pas viser à

10 Voir AESH, DP-57M, M. Ed. Crevier à Mgr J.-C. Prince (12 avril 1853).

11 Voir APM, Histoire de Marieville par le Chan. J.-B.-A. Allaire, 1924, revilue, corrigée et augmentée en 1939, par le Chan. J.-B.-O. Archambault.

12 AESH, DP-57M, M. Ed. Crevier à Mgr J.-C. Prince (7 août 1853).

13 AESH, RL, Série I, v. 1, Mgr J.-C. Prince à M. Ed. Crevier (9 août 1853).

14 Ibid. 
devenir un Collège classique et encore moins un Petit Séminaire. $\mathbf{M}^{* r}$ Prince aurait voulu y voir implanter un cours de cinq années précédé d'une année préparatoire pour ceux «qui n'auraient pas une connaissance suffisante en langue française ${ }^{15}$ au niveau de la lecture et de l'écriture. Les matières à l'étude durant ce cours auraient été les langues française et anglaise, les mathématiques, la géographie, l'histoire, l'agriculture. la tenue des livres, la correspondance «marchande et d'affaires », le dessin linéaire, l'économie domestique, la chimie et quelques notions générales de rhétorique et de philosophie ${ }^{16}$. L'évêque ne parle pas des langues grecque et latine. Pour lui, le Collège de Sainte-Marie-de-Monnoir ne doit vraiment être qu'un Collège ou une Académie, c'est-à-dire une maison d'enseignement se situant entre le primaire et le Collège classique.

Énergique et entreprenant, renonçant difficilement à ses idées, M. Crevier ne se laisse pas impressionner par les désirs de son évêque. D'ailleurs, M. Crevier recevra plusieurs fois des reproches de ses supérieurs hiérarchiques à ce propos, $\mathrm{M}^{\mathrm{wr}}$ Charles LaRocque lui reprochant même de vouloir jouer «à l'évêque ${ }^{17}$.

Le plan d'études que M. Crevier propose pour le Collège SainteMarie-de-Monnoir est différent de celui qui est habituellement suivi dans les collèges classiques ou petits séminaires, notamment celui de Saint-Hyacinthe. Le plan qu'il propose tient compte d'une réalité concrète de l'époque : beaucoup de jeunes commencent leur collège mais doivent, pour plusieurs raisons, abandonner après un certain nombre d'années; ou encore, entrevoyant d'avance l'impossibilité pour eux de terminer le cours complet et d'entreprendre ensuite la formation qui conduirait à l'une ou l'autre des professions libérales, ils préfèrent ne pas poursuivre d'études après le primaire. Dans l'optique de M. Crevier, les jeunes doivent avoir la possibilité de poursuivre, dans la mesure où leurs moyens financiers le leur permettent, des études supérieures, et d'en retirer «la plus grande somme d'avantages relativement au nombre d'années passées dans le Collège $\gg 18$.

15 AESH, DP-57M, Plan d'éducation all Collège de Sainte-Marie-deMonnoir (1858).

16 Ibid.

17 AESH, RL, Série I, Belœil, v. 1, Mgr C. LaRocque à M. Ed. Crevier (17 arril 1869); voir aussi AESH, RL, Série I, v. 1, Mgr J.-C. Prince à $M$. Ed. Crevier (9 âtut 1853); Mgr J.-C. Prince à M. Ed. Crevier (16 juin 1855); RL, Série I, v. 4, Mgr J. Larocque à M. Ed. Crevier (26 aô̂t 1853); Mgr $J$. Larocque à M. Ed. Crevier (7 mars 1864).

18 Le Courrier de Saint-Hacinthe, 6 septembre 1853. 
Pour cela, il faut offrir aux jeunes Canadiens français une éducation pratique, une "éducation dont toutes les parties quelque peu étendues qu'elles soient, puissent se coordonner ensemble de manière à devenir toutes utiles à celui qui les possèdera à un degré quelconque »19. Pour M. Crevier ce n'est donc pas la quantité, la somme ou l'étendue des connaissances qui importe, mais bien leur utilité pour l'avenir. De plus, les maisons d'éducation supérieure devraient viser à donner, et cela d'une façon particulière au peuple canadienfrançais, une éducation qui lui permette à lui aussi de «travailler à augmenter notre prospérité conjointement avec ses autres compatriotes » ${ }^{20}$. D'où le fait que, dans son plan d'études, M. Crevier accorde autant d'importance à l'anglais et aux matières qui se rapportent au monde du commerce et des affaires qu'aux langues et aux disciplines conduisant aux professions libérales et ecclésiastiques. Pour lui, une maison d'enseignement supérieur doit viser autant à la formation d'hommes, de citoyens qui soient capables d'agir à tous les niveaux et dans tous les secteurs qui composent la vie concrète d'une scciété, qu'à celle de professionnels et d'ecclésiastiques.

Pour arriver à cela, M. Crevier propose un plan d'études qui se divise en trois étapes successives. La première se compose de quatre années. Elle offre une formation aux sciences mathématiques, à la géographie et à l'histoire, aux langues française et anglaise, et se termine, la quatrième année, par l'étude de la mécanique, du dessin linéaire, de l'agriculture, du commerce, de la tenue des livres et de l'économie domestique. Cette première étape se caractérise par l'importance accordée à l'anglais.

La méthode pédagogique pour l'enseignement des matières telles que les langues, l'histoire et la géographie, se veut également nouvelle. Afin de permettre à l'étudiant de tirer le plus d'avantages possibles des années d'études qu'il fera, on commence par lui enseigner ce qui lui sera le plus utile, ce qui est le plus proche de lui au moment où il se présente pour commencer le cours d'études. Ainsi, par exemple, on commence par lui enseigner l'histoire de son pays en première et deuxième années; en troisième ce sera l'histoire de l'Angleterre et de la France, les deux mères patries, et en quatrième, l'histoire moderne suivie de l'histoire du Moyen Âge. C'est donc une méthode pédagogique qui remonte dans le temps. Un étudiant n'apprendra l'histoire de l'Antiquité que s'il suit le cours complet de

20 Ibid. 
huit ans. En adoptant cette méthode de présentation des différentes matières au programme, on offrait donc aux jeunes un avancement progressif à rebours si l'on veut, mais qui leur était utile s'ils ne pouvaient suivre que trois ou quatre années d'études. Car il n'y a pas de doute que pour un jeune Canadien français du XIX" siècle qui ne peut se payer le luxe de suivre un cours d'études de huit années, la connaissance de l'histoire de son pays est plus utile, plus avantageuse et plus pratique que la connaissance de l'histoire de l'Antiquité classique grecque ou romaine.

Nous retrouvons la même méthode appliquée à l'apprentissage de l'anglais. Le plan prévoit une connaissance pratique de cette langue par le dialogue et la conversation pour la deuxième année, suivie en troisième, de l'étude des règles de grammaire. Ainsi, l'étudiant qui ne pouvait suivre que deux années d'études, pouvait au moins parler anglais même s'il ne savait pas toutes les règles de grammaire.

Cette première partie du plan d'études de M. Crevier correspondait assez bien aux vues de $\mathbf{M}^{\mathrm{gr}}$ Prince sur la maison d'éducation qui devait être établie à Sainte-Marie-de-Monnoir. Là où les deux divergeaient d'opinion, c'était pour les quatre années suivantes que proposait le curé.

Cette deuxième section du plan de $\mathrm{M}$. Crevier devait être consacrée à l'étude des matières du cours classique telles que les langues grecque et latine, la littérature et la philosophie. Encore ici, nous retrouvons les mêmes principes d'enseignement et de répartition des matières. Cette section se divisait en deux parties qui se voulaient complètes par elles-mêmes: les deux dernières années étant un approfondissement de ce qui avait été vu dans les deux premières années de cette section dite classique. Enfin, la dernière année comprenait également une revue générale de l'ensemble du cours d'études.

Ce plan d'études, M. Crevier le modifiera en 1859. Le nouveau plan d'études qu'il proposera alors comprendra trois sections: la première, d'une durée de trois ans, était consacrée au commerce, à l'agriculture, ainsi qu'aux arts et métiers; la deuxième préparait les jeunes qui voulaient poursuivre une profession libérale, et la troisième était destinée à «former à l'État Ecclésiastique ou à préparer aux hautes fonctions de la vie publique $\gg 21$.

21 Voir AESH, DP-57M, Plan du cours d'études du Collège SainteMarie-de-Monnoir, par M. E. Crevier (28 juin 1859). 
Un élève qui arrivait suffisamment préparé en français et en mathématiques pouvait donc faire le cours complet en sept ans. Cette formule ne variera pratiquement pas, sauf qu'à un certain moment, - malheureusement, nous n'avons pu retracer l'année exacte - le cours préparatoire disparut et le cours classique fut porté à cinq ans au lieu de quatre. Le cours complet comprendrait désormais huit années d'études: trois pour le cours commercial, et cinq pour le cours classique. Cette formule reste inchangée jusqu'à la disparition du Collège en 1912.

Même si M. Crevier n'envisageait pas de mettre sur pied, dès l'ouverture du Collège, le plan complet des huit années du cours d'études, il lui fallait quand même recruter le personnel nécessaire pour démarrer. C'est ainsi que le 12 avril 1853, il demandait à son évêque un prêtre et deux ecclésiastiques pour la direction du Collège, la «conduite des classes et la garde des élèves $2^{22}$. Il proposa même à $\mathrm{M}^{\mathrm{w}}$ Prince de lui adjoindre comme directeur du Collège, l'abbé Isidore Desnoyers qui avait été son vicaire à Saint-Hyacinthe de 1846 à 1852. L'évêque lui répondit qu'il n'avait pas d'objection à ce que M. Desnoyers le rejoigne à Sainte-Marie-de-Monnoir pour prendre la direction du Collège, mais qu'avant tout, il lui fallait "préalablement pourvoir aux cures et aux missions $\gg 2.3$ du diocèse. M. Desnoyers n'ira jamais à Sainte-Marie-de-Monnoir. De fait, M. Crevier devra se contenter du personnel qu'il avait déjà près de lui. L'abbé Olivier Désorcy, son vicaire, remplira la charge de directeur du Collège durant la première année.

En fait, à chaque année, M. Crevier devra se préoccuper de trouver du personnel pour son Collège. Ccux qu'il réussira à intéresser à son œuvre, demanderont toujours, après un an ou deux, de quitter Sainte-Marie-de-Monnoir pour aller en paroisse ou encore aller auvrer en Nouvelle-Angleterre. Ce n'est que vers les années 1864-1865, que le Collège, quant à son personncl directif et enseignant, acquerra une certaine stabilité grâce aux premiers ecclésiastiques qui, ayant fait leurs études au Collège, voudront travailler à son développement et son épanouissement. Ce sont des étudiants de la première heure, MM. Joseph-Alfred Nadeau, Jean-Baptiste Ponton ct Isidore Bessette.

22 AESH, DP-57M, M. Edi. Crevier à Mgr J.-C. Prince (12 avril 1853).

23 AF.SH. RL. Série I, v. I, Mgr J.-C. Prince à M1. Ed. Crevier (14 (avil 1853). 
L'entretien matériel de la maison, ainsi que le service auprès des prêtres, des ecclésiastiques et des étudiants, seront assurés par «des domestiques des environs, des personnes âgées » ${ }^{24}$, jusqu'à ce que les Petites Sœurs de la Sainte-Famille de Sherbrooke les remplacent au cours de l'année $1894^{25}$. En 1904, la Congrégation des Frères de Saint-Gabriel acceptait d'envoyer cinq de ses religieux au Collège Sainte-Marie-de-Monnoir ${ }^{26}$. Malheureusement, ces deux communautés, à cause des conditions matérielles qui leur étaient imposées après l'incendie de 1907 et le refus des autorités du Collège de reconstruire, décidèrent de se retirer du Collège Sainte-Marie-de-Monnoir à la fin de l'année académique de 1907-1908 ${ }^{27}$.

De 1853 à 1867, M. Crevier vit grandir et s’affermir son œuvre. Un an après l'ouverture du Collège, il avait entrepris des démarches en vue d'obtenir l'incorporation civile. Celle-ci lui fut accordée le 3 avril $1855^{28}$. La nouvelle corporation portait le nom de «La corporation du Collège de Monnoir » et se composait «du prêtre et du curé de la dite paroisse de Sainte-Marie-de-Monnoir, du marguillier en exercice de l'œuvre et fabrique de l'église de la susdite paroisse, du principal et du procureur du dit collège, du préfet des études. et de leurs successeurs en office ${ }^{29}$. Le Collège de Sainte-Marie-deMonnoir se trouvait donc, à cause des membres qui en composaient la corporation, - le curé, le vicaire et le marguillier en exercice étroitement lié à la paroisse.

Après 1865, le Collège de Sainte-Marie-de-Monnoir avait un cours d'études bien déterminé, ses objectifs étaient bien connus. et il dispensait son enseignement à près de 100 étudiants. Mais une réalité plus importante laissait croire à une plus grande stabilisation de cette jeune institution et pouvait laisser espérer en son avenir : depuis 1864-1865, deux des premiers élèves du Collège, les abbés Jean-Baptiste Ponton et Joseph-Alfred Nadeau. dès la fin de leurs études, avaient manifesté le désir de se joindre au personnel respon-

24 AESH, DP-57M, M. Ed. Crevier à Mgr J.-C. Prince (7 uoût 1853).

25 Voir AESH. RL, Série I. v. 18, M!gr L.-Z. Morcall à M. J.-A. Lemieux (26 novembre 1895).

26 Voir ASSH. FSSM, FG-43, 32, 26, Annucire du Collège SuinteMaric'-de-Monnoir, 1904-1905.

27 Voir AESH, RL, Série II, v. 2, Mgr L.-Z. Morecul à M. J.-A. Lemieux (14 juin 1908).

28 Voir ASSH, FSMM, FG-43, 32, 13. Acte pour incorporer le Collège de Monnoir (sanctionné le 3 arril 1855).

29 Ibid. 
sable de leur Alma Mater. L'évêque d'alors, $\mathbf{M}^{\mathrm{gr}}$ Joseph Larocque, avait acquiescé à leur demande. Le Collège de Monnoir venait donc de franchir une étape importante de son existence : l'auto-suffisance dans le recrutement de son personnel.

Le grand rêve de $\mathbf{M}$. Crevier était de voir un jour son Collège reconnu comme Petit Séminaire. En 1867, M. Crevier et les prêtres du Collège crurent ce moment arrivé. Ils écrivirent donc à l'évêque, $\mathrm{M}^{\mathrm{wr}}$ Charles LaRocque, pour lui demander de bien vouloir ériger canoniquement le Collège Sainte-Marie-de-Monnoir en Petit Séminaire. Ce que $\mathrm{M}^{\mathrm{rr}}$ Charles LaRocque fit en publiant un mandement le 15 septembre $1867{ }^{30}$.

Dans ce Mandement, l'évêque déclarait reconnaître le Collège de Monnoir comme un petit séminaire diocésain où des prêtres de son diocèse se dévouaient à l'éducation des jeunes en général, mais plus particulièrement de ceux appelés au service de l'Église et de l'autel. Il y établissait également une corporation ecclésiastique qui se composerait de tous les prêtres qui obtiendraient la permission de l'évêque de s'agréger au Petit Séminaire. Cette corporation était reconnue sous le nom des «Prêtres du Petit Séminaire de SainteMarie, lesquels, en toute chose et en tous les temps, seront et demeureront sous l'entière juridiction de l'Évêque diocésain ${ }^{31}$.

Au plan civil, la charte d'incorporation civile de 1855 était respectée dans toute son étendue ${ }^{32}$. Notons ici, en passant, qu'en 1867 , les membres de la Corporation civile et les membres de la Corporation ecclésiastique n'étaient pas les mêmes puisque la Corporation civile comptait parmi ses membres le curé, le vicaire et le marguillier en exercice, alors que la Corporation ecclésiastique ne regroupait que M. Crevier et les prêtres directement engagés dans la direction du Collège. Le Collège ou Petit Séminaire de Sainte-Marie-de-Monnoir se trouvait donc soumis à deux corporations composées de personnes différentes, sauf pour M. Crevier.

En 1855, M. Crevier avait entrepris d'agrandir le Collège. Il escomptait d'être capable, avec les octrois gouvernementaux ainsi qu'avec les dons généreux de son frère aîné, l'abbé Joseph Crevier,

30 Voir Mandements, lettres pastorales et circulaires des Évêques de Saint-Hyacinthe, t. 2, p. 454-460.

$31 \quad$ Ibid., p. 456.

32 Ibid., p. 457. 
de défrayer les coûts de l'agrandissement entrepris. Malheureusement, ceux-ci furent plus élevés qu'il avait prévu. Il semble bien qu'après avoir réussi à monter les quatre étages et y avoir placé un toit, M. Crevier ait dû renoncer à poursuivre les travaux. Au plan matériel, selon les prêtres du Collège eux-mêmes, le Collège était dans un état lamentable et les conditions de vie étaient pratiquement intenables. Au plan financier, aux dires de ces derniers, le Collège était sérieusement menacé par la banqueroute.

En 1869, M. Crevier pensa agrandir le Couvent des filles en utilisant l'argent que son frère Joseph avait déclaré formellement vouloir laisser au Petit Séminaire à sa mort. Les prêtres de Monnoir écrivirent donc un long mémoire à $\mathrm{M}^{*}$ Charles LaRocque pour $\mathrm{y}$ dénoncer la situation du Collège au plan matériel et financier, et pour lui demander d'interdire à $M$. Crevier la poursuite de ses projets pour le Couvent des Sœurs ${ }^{33}$. $\mathrm{M}^{\mathrm{wr}}$ LaRocque intervient auprès de M. Crevier pour lui demander de ne pas s'engager dans de nouveaux projets au détriment du Collège. De plus, il envoya l'abbé Louis-Zéphirin Moreau faire une enquête pour connaître la véritable situation financière du Collège, et demanda à ce que des Constitutions répartissant les tâches et les responsabilités au sein des membres de la Corporation ecclésiastique lui soient présentées pour être approuvées.

Cette crise au sein des membres du personnel du Petit Séminaire de Sainte-Marie-de-Monnoir fut bien près de signifier sa disparition. Mais grâce à la volonté et à l'attachement des prêtres de Monnoir à leur Alma Mater, le Petit Séminaire put être sauvé. Cependant, cette crise eut pour effet de faire connaître l'extrême précarité de la situation financière de l'institution, sa dette totale s'élevant à plus de $\$ 6,500.00$ à la fin de l'année académique 1868-1869. De plus, elle permit de bien déterminer les tâches et les responsabilités au sein de la Corporation ecclésiastique, chose qui n'avait pas été faite au lendemain de l'érection canonique du Collège en Petit Séminaire.

Au début des années 1870. devant l'augmentation toujours croissante des inscriptions, les autorités sentirent le besoin d'agrandir le Collège. C'est ainsi qu'en 1873, les membres de la Corporation

33 Voir AESH, DP-57M, Mémoire des prêtres du Petit Séminaire de Sainte-Marie-de-Monnoir concernant les atjaire's temporelles de la maison (21 mars 1869). 
avaient décidé l'agrandissement du Petit Séminaire ${ }^{34}$. Mais à cause de la situation financière dans laquelle se trouvait l'institution, ils retardèrent l'exécution de leur projet pour quelques années. À l'automne de 1875, alors que le Petit Séminaire comptait 206 étudiants, soit une augmentation de $10 \%$ par rapport à l'année précédente, les membres de la Corporation décidèrent de demander à $\mathbf{M}^{\mathrm{yr}}$ LouisZéphirin Moreau, nouvel évêque-élu de Saint-Hyacinthe depuis le 19 novembre 1875 , les permissions nécessaires ${ }^{35}$. Pour aider à défrayer les coûts de cet agrandissement, la Fabrique de Sainte-Marie-deMonnoir s'engagea, le 9 janvier 1876, à verser un don de $\$ 2,000.00$ à la Corporation du Petit Séminaire ${ }^{36}$. Devant la générosité des paroissiens de Sainte-Marie, $\mathbf{M}^{\mathrm{gr}}$ Moreau accorda les permissions demandées.

Cependant, $\mathbf{M}^{\mathrm{gr}}$ Moreau, étant au courant de la véritable situation et des problèmes du Petit Séminaire de Sainte-Marie-de-Monnoir par suite de l'enquête que l'évêque lui avait demandé de faire au moment de la crise de 1869, avait son opinion sur le Petit Séminaire et son personnel. C'est ainsi que le 15 juillet $1876, \mathrm{M}^{\mathrm{gr}}$ Moreau nommait l'abbé François-Xavier Jeannotte «Vice-supérieur du Petit Séminaire de Sainte-Marie-de-Monnoir» avec la mission de veiller à «la direction générale et particulière » du Collège et de "surveiller tous les offices de la maison et ceux qui les exercent ${ }^{37}$. Et il écrivait à M. Crevier :

En augmentant le personnel de votre Collège, je ne dois pas vous laisser ignorer, Mr. le G. Vicaire qu'une de mes premières intentions a été de vous décharger des détails de la régie intérieure de l'institution, et d'accoutumer peu à peu les Prêtres qui y demeurent, à se suffire à eux-mêmes et à se conduire par eux-mêmes, tout en ayant soin cependant de recourir à vous ou à l'Évêque dans les affaires embarrassantes ou dans les cas extraordinaires 38 .

Enfin, le 30 juillet $1877, M^{\text {gr }}$ Moreau écrivait de nouveau à M. Crevier pour lui dire qu'il était déterminé à envoyer M. Joseph-

34 Voir AESH, DP-57M, M. Ed. Crevier à Mgr L.-Z. Moreau (30 décembre 1875).

35 Ibid.

36 Voir AESH, DP-57M. M. Ed. Crevier à Mgr L.-Z. Moreau (18 janvier 1876).

37 AESH, RL, Série I, v. 7, Mgr L.-Z. Moreau à M. F.-X. Jeannotte (15 juillet 1876).

38 AESH, RL, Série I, v. 7, Mgr L.-Z. Moreau à M. Ed. Crevier (25 août. 1876). 
Alfred Nadeau, alors procureur du Collège, au ministère curial. Dans sa lettre, il lui rappelait qu'il lui «paraissait essentiel que les premiers éléments de la maison disparaissent pour faire place à un personnel tout nouveau et animé d'un esprit tout différent ${ }^{39}$. Avec le départ de M. Nadeau, nous assistons au départ du dernier membre de l'équipe qui s'était constituée au milieu des années 1860 , et qui avait vécu la crise de 1869 .

Pendant ce temps, $\mathrm{M}^{\mathrm{gr}}$ Moreau avait envoyé l'abbé Alexis-Xyste Bernard au Petit Séminaire de Sainte-Marie-de-Monnoir afin d'étudier la situation financière de l'institution ${ }^{40}$. Selon le rapport de l'abbé Bernard et celui de l'abbé Jeannotte, la dette totale du Collège s'élevait à plus de $\$ 17,000.00$. De plus, de cette somme, il y avait un montant de $\$ 13,446.26$ qui portait intérêt à $7 \%$, soit $\$ 921.26$ par année. Le Petit Séminaire de Sainte-Marie-de-Monnoir se trouvait donc dans une situation financière fort embarrassante : si des mesures efficaces de redressement n'étaient pas prises immédiatement, il était menacé de faillite dans un avenir plus ou moins rapproché.

Les autorités du Collège se mirent donc à la recherche d'une solution possible. M. Crevier proposa d'abandonner la cure de SainteMarie-de-Monnoir en faveur du Petit Séminaire ${ }^{41}$. Cette solution permettrait au Collège de liquider sa dette en neuf ans. Devant des promesses de résultats aussi prometteurs, $\mathbf{M}^{\mathrm{gr}}$ Moreau, malgré la réticence qu'il éprouvait à confier une cure à une communauté de prêtres telle que celle formée par les prêtres du Petit Séminaire de Sainte-Marie-de-Monnoir, écrivait à $\mathrm{M}$. Crevier pour lui demander s'il était toujours prêt à se désister de sa cure en faveur du Petit Séminaire ${ }^{42}$. Le 27 septembre 1877 , M. Crevier lui répondit affirmativement. C'est ainsi que M. Jeannotte reçut ses lettres testimoniales comme curé de Sainte-Marie-de-Monnoir le 30 novembre 1877. Pour sa part, M. Crevier, tout en conservant les facultés de Grand Vicaire du diocèse et son titre de Supérieur du Petit Séminaire de Sainte-Marie-de-Monnoir, se retira à l'Hospice Sainte-Croix de SainteMarie-de-Monnoir où il devait décéder le 22 janvier 1881.

39 AESH, RL, Série I, v. 7, Mgr L.-Z. Moreau à M. Ed. Crevier (30 juillet 1876).

40 Voir AESH, DP-57M, Rapport de l'examen des finances du Collège Ste-Marie fait par Mr. A.-X. Bernard, ass. secrétaire de Mgr L.-Z. Moreau, évêque de Saint-Hyacinthe (février 1877).

41 Voir AESH, DP-57M, M. F.-X. Jeannotte à Mgr L.-Z. Moreau (4 novembre 1877).

42 Voir AESH, RL, Série I, v. 8, Mgr L.-Z. Moreau à M. Ed. Crevier (23 novembre 1877). 
Cette solution fut-elle efficace ? Le 5 novembre 1882, M. Jeannotte écrivait à $\mathbf{M}^{\mathrm{gr}}$ Moreau que la dette du Collège était maintenant à $\$ 8,600.00$, et que si tout continuait à bien aller, il pensait pouvoir donner $\$ 100.00$ d'honoraires à tous les prêtres du Collège. Or, avant d'adopter cette solution, on avait estimé qu'en 1882, la dette ne serait plus que de $\$ 9,592.67^{43}$. Le nouvel état de choses établi en 1877 avait donc permis de verser près de $\$ 1,000.00$ de plus que prévu.

Devant les résultats plus que positifs de la solution mise de l'avant depuis 1877, $\mathrm{M}^{\mathrm{gr}}$ Moreau, le 9 juin 1884, écrivit au Pape Léon XIII pour lui demander la permission d'unir à perpétuité les revenus de la dîme et du casuel de la Paroisse de Sainte-Marie-deMonnoir au Petit Séminaire établi dans la même paroisse. Il expliquait au Pape que son intention était uniquement de fournir à cette institution une source de revenus supplémentaires stable qui lui permettrait de faire les améliorations et les réparations nécessaires qu'exigerait ce genre d'institution si elle désirait se "mettre au niveau des besoins et des exigences du temps et faire son œuvre d'une manière plus efficace ${ }^{44}$. L'indult établissant à perpétuité le Petit Séminaire de Sainte-Marie-de-Monnoir curé de la paroisse du même nom, fut obtenu le 8 mars 1885 .

Le Petit Séminaire de Sainte-Marie-de-Monnoir se trouvait ainsi placé dans une situation unique en tant que maison d'enseignement supérieur. Il fut sans doute le seul établissement d'enseignement de la Province de Québec ayant la responsabilité directe d'une paroisse.

$\mathrm{M}^{* r}$ Moreau voulut également régulariser la situation en ce qui concernait les Corporations civile et ecclésiastique et aussi les Constitutions qui régissaient la vie du Petit Séminaire de Sainte-Marie-deMonnoir. Aussi commença-t-on par demander à la Législature de Québec un acte amendant l'Acte d'incorporation civile de 1855. Dorénavant, le curé, le vicaire et le marguillier en exercice étaient exclus de la Corporation civile dont le nom devenait «La corporation du Petit Séminaire de Ste-Marie-de-Monnoir». De plus, à l'article quatrième on ajoutait :

L'évêque de Saint-Hyacinthe aura le droit de voter lorsqu'il sera présent aux délibérations du dit conseil de La corpo-

43 Voir AESH, DP-57M, Les prêtres de la Corporation de Monnoir à Mgr L.-Z. Moreau (fin 1877).

44. Voir AESH, RL, Série I, v. 12, Mgr L.-Z. Moreau au Pape Léon XIII (9 juin 1884). 
ration du petit séminaire de Sainte-Marie-de-Monnoir, qu'il pourra présider ${ }^{45}$.

Il ressort donc de l'Acte de 1880 , que les liens avec la paroisse étaient coupés. Désormais, la Corporation du Collège Sainte-Mariede-Monnoir était indépendante des liens qu'elle avait eus jusqu'à maintenant avec la cure et la Fabrique de Sainte-Marie-de-Monnoir. L'évêque de Saint-Hyacinthe, nous dit également l'Acte de modification, pourra, lorsquil assistera aux réunions, présider et voter. Mais ce ne sont là que des concessions respectueuses ou des privilèges qu'on lui accorde parce qu'il est l'autorité diocésaine et qu'il se trouvera sur les lieux. De droit l'évêque de Saint-Hyacinthe n'était pas membre de la Corporation civile du Petit Séminaire de SainteMarie-de-Monnoir. Cela peut nous étonner, surtout que depuis le 15 septembre 1867, $\mathrm{M}^{\mathrm{gr}}$ Charles LaRocque, évêque de Saint-Hyacinthe, a érigé canoniquement le Collège Sainte-Marie-de-Monnoir en Petit Séminaire. Or, il y a un autre Petit Séminaire dans le diocèse de Saint-Hyacinthe, celui de la ville épiscopale, dont l'évêque est non seulement membre de la Corporation, mais président de celle-ci, bien qu'il n'en ait pas été ainsi dès le début. Ainsi, la position de l'évêque diocésain au plan de la juridiction civile en ce qui concerne le Collège Sainte-Marie-de-Monnoir, bien que ce dernier soit érigé en Petit Séminaire depuis 1867, était différente de celle qu'il occupait vis-à-vis le Petit Séminaire de sa ville épiscopale.

Après l'indult de 1884 établissant le Petit Séminaire de SainteMarie-de-Monnoir curé de la paroisse, $\mathrm{M}^{\mathrm{gr}}$ Moreau crut le moment venu de refaire les Constitutions du Petit Séminaire. L'Acte de 1880 amendant l'Acte d'incorporation de 1855, concédait à la Corporation du Petit Séminaire le droit de faire des règlements et des statuts. Aussi, le 21 mai 1884, M $^{*}$ Moreau réunit-il les prêtres du Petit Séminaire pour renouveler les Constitutions de leur institution ${ }^{46}$. À l'occasion de la refonte de leurs Constitutions, les prêtres de Monnoir repensèrent les différents niveaux d'administration, la répartition des tâches, les conditions d'agrégation, et insérèrent dans les nouvelles Constitutions un article qui soumettait le temporel du

45 Voir ASSH, FSSM, FG-43, 32, 12, Acte pour changer le nom du "Collège de Monnoir» en celui de "Petit Séminaire de Ste Marie de Monnoir» et pour autres fins (sanctionné le 24 juillet 1880).

46 Voir ASSH, FSSM, FG-43, 32, 28, Nouvelle constitution du Petit Séminaire de Sainte-Marie-de-Monnoir (21 mai 1884). 
Collège à la jurdiction de l'évêque de Saint-Hyacinthe, lequel était déclaré membre à part entière de la Corporation.

Il ne sera jamais permis d'engager les biens du Petit Séminaire en caution pour quelque personne que ce soit. Chaque fois qu'il sera question d'aliénation, il sera toujours nécessaire de faire approuver la transaction par Monseigneur l'évêque 47.

Ainsi, les ambiguïtés et les équivoques au niveau des juridictions civile et ecclésiastique se trouvaient donc supprimées.

Le supériorat de $\mathrm{M}$. Jeannotte se termina en 1894 , alors que M. Joseph-Alfred Lemieux lui succéda à la tête du Petit Séminaire. De 1884 à 1894, le Collège Sainte-Marie-de-Monnoir, grâce aux attentions et la bonne administration de $\mathrm{M}$. Jeannotte, connut la période la plus florissante de son histoire. Sa renommée s'étendit partout et le nombre de ses étudiants ne cessait d'augmenter : au milieu des années 1890 , le nombre total des inscriptions atteignait presque 250 , et de ce nombre $33 \%$ étaient d'origine américaine. C'est également sous le supériorat de $M$. Jeannotte que le Petit Séminaire de Sainte-Marie-de-Monnoir demanda officiellement son affiliation à l'Université Laval, le 25 décembre 187948 . Enfin, nous pouvons ajouter qu'en 1886, le Collège dut agrandir ses locaux de façon assez considérable si l'on considère que le coût des travaux s'éleva à $\$ 26,000.0049$.

Le 18 mai $1894, M^{\text {gr }}$ Moreau avisa $M$. Jeannotte qu'il acceptait sa démission comme supérieur du Petit Séminaire de Sainte-Maric-deMonnoir. Les prêtres du Collège se réunirent donc pour procéder à l'élection d'un nouveau supérieur comme le leur en donnaient le droit les Constitutions de 1884. Ils élirent l'abbé Joseph-Alfred Lemieux, professeur de philosophie. Sur réception des résultats, $\mathbf{M}^{\mathrm{rr}}$ Moreau s'empressa de confirmer $M$. Lemieux dans ses nouvelles fonctions de curé et de supérieur du Petit Séminaire de Sainte-Mariede-Monnoir ${ }^{50}$. Le supériorat de $\mathbf{M}$. Lemieux en fut un des plus agités. Il n'avait que 37 ans lors de son élection à la tête du Petit

47 Ibid.

48 Voir APSQ, UL, 130-BI, Mgr J.-S. Raymond à M. T.-E. Hamel (9 février 1880).

49 Voir AESH, DP-57M, M. F.-X. Jeannotte à Mgr L.-Z. Moreau (14 aô̂t 1893).

50 Voir AESH, RL, Série I, v. 17, Mgr L.-Z. Moreau à M. V. Larose (19 juin 1894). 
Séminaire, et il apportait avec lui des idées neuves. Son désir était de faire du Petit Séminaire un Collège moderne et d'avant-garde.

Les locaux qu'occupait le Petit Séminaire étaient en mauvais état; seule la dernière construction de 1886 était encore dans un état satisfaisant. La première étape était donc de reconstruire. Mais comme la question financière avait toujours été la pierre d'achoppement de cette institution, il fallait donc trouver une solution à ce problème avant même de faire quoi que ce soit. À cette époque, le Gouvernement fédéral encourageait le développement des terres de l'Ouest du pays. M. Lemieux, ainsi que quelques amis influents du Petit Séminaire, eurent l'idée de demander au Gouvernement fédéral la permission de fonder une société de colonisation qui verrait à encourager des Canadiens français à aller s'installer dans l'Ouest du pays ${ }^{51}$. Ils demandèrent une concession et ils l'obtinrent en avril 1900. Cette concession se trouvait à la Rivière-de-la-Paix en Alberta ${ }^{52}$. Les membres de cette Société s'étaient tous engagés à verser au Petit Séminaire les profits de la Société afin de lui assurer les argents nécessaires à la reconstruction.

En 1905, il se présenta une occasion unique pour la Société. En effet, un certain Ernest $\mathrm{H}$. Kent voulut se porter acquéreur des terres qui avaient été concédées à la Société de Colonisation. Il en offrait $\$ 100,000.00$. Devant une offre aussi généreuse, les membres de la Société décidèrent de vendre, et le contrat fut signé le 23 septembre $1905{ }^{53}$. Les prêtres de Monnoir se retrouvaient donc en possession d'une somme assez importante pour pouvoir penser sérieusement à reconstruire le Petit Séminaire. Ils se réunirent donc pour en discuter et arrivèrent à la conclusion que, vu la nature du sol, l'incapacité de l'aqueduc et l'insalubrité des égouts, il valait mieux reconstruire ailleurs ${ }^{54}$. M. Lemieux et $\mathrm{M}$. Bergeron, procureur du Collège, se rendirent à Saint-Hyacinthe présenter leur requête au nouvel évêque, $\mathrm{M}^{\mathrm{gr}}$ A.-X. Bernard.

51 Voir ASSH, FSSM, FG-43, 32, 21, Déclaration des membres de la Société de Colonisation de la Rivière-de-la-Paix (12 juin 1907).

52 Voir APC, Sir Wilfrid Laurier, MG-26-G, vol. 150, n" +441 1-44412. M. Louis-Philippe Brodeur à Sir Wilfrid Laurier (6 alril 1900).

53 Voir ASSH, FSMM. FG-43, 32. 14, Bref du Quo Warranto émis par le juge Dominique Monet de la Cour supérieure du District d'Iberville (15 juillet 1906).

54 Voir ASSH, FSMM, FG-43, 32, 15, Procès-verbal de la réunion des prêtres de la Corporation du Petit Séminaire de Sainte-Marie-de-Monnoir (19 mars 1906). 
Devant la requête des prêtres du Petit Séminaire, $\mathbf{M}^{\mathrm{gr}}$ Bernard demanda à ce que les allégations sur la nature du sol, l'aqueduc et les égouts soient confirmées par des experts. Dans leurs rapports, les trois experts reconnaissaient le bien fondé des allégations des prêtres du Petit Séminaire et que celles-ci représentaient des difficultés quasi insurmontables ${ }^{55}$. Devant les rapports des experts, et après avoir consulté son Chapitre, $\mathrm{M}^{\mathrm{gr}}$ Bernard accorda aux prêtres du Petit Séminaire de Sainte-Marie-de-Monnoir la permission de reconstruire leur institution dans la localité de Richelieu ou sur les bords de la rivière Richelieu. Cette permission leur fut accordée le 10 novembre $1906^{56}$.

L'année 1907 s'annonçait donc comme une année importante et décisive pour le Petit Séminaire de Sainte-Marie-de-Monnoir. Ayant reçu la permission de $\mathrm{M}^{\mathrm{gr}}$ Bernard de reconstruire ailleurs leur Collège, les prêtres de Monnoir devaient donc essayer maintenant de déterminer s'ils reconstruiraient au village de Richelieu ou ailleurs. Malheureusement, les joies et les espérances nées de la permission épiscopale allaient bientôt être assombries par le drame, et le Petit Séminaire de Sainte-Marie-de-Monnoir allait connaître les heures les plus difficiles de son histoire.

Le 23 février 1907, le Petit Séminaire était la proie des flammes: il fut détruit de fond en comble. C'est vers 10.30 heures du matin que le «feu se déclara dans les dortoirs par une cheminée défectueuse, que la cuisine avait surchauffée $\gg 57$. Vers 3.00 heures de l'après-midi, tout était terminé : le Petit Séminaire de Sainte-Mariede-Monnoir qui existait depuis plus de 50 ans, "n'était plus que des ruines ${ }^{58}$. La nouvelle du malheur qui venait de frapper le Collège se répandit rapidement. M. Lemieux et les prêtres de Monnoir reçurent de nombreux témoignages de sympathie. Au moment de l'incendie, le Petit Séminaire abritait plus de 280 personnes : 242 étudiants, 20 prêtres séculiers, 15 religieuses de la Sainte-Famille de Sherbrooke et 11 frères de Saint-Gabriel.

55 Voir ASSH, FSSM, FG-43, 32, 12, M. Maurice Perrault à M. J.-A. Lemieux (12 mai 1906); FSMM, FG-43, 32, 20, M. Joseph Haynes à. M. J.-A. Lemieux (mai 1906); Wm. Hood \& Son à M. Maurice Perrault (mai 1906).

56 Voir ASSH, FSMM, FG-43, 32, 12, Mgr A.-X. Bernard à M. J.-A. Lemieux (10 novembre 1906).

57 ASCH,Chroniques de l'Hospice Sainte-Croix, Marieville, 1893-1908.

58 Ibid. 
Après bien des démarches, les prêtres de Monnoir réussirent à poursuivre leur enseignement. Les Sœurs de la Charité, après avoir transporté les pensionnaires dans leurs autres maisons ou chez des particuliers, mirent leur Hospice à la disposition des autorités du Collège. Les Frères de Saint-Gabriel trouvèrent refuge à la maison Franchère, et les Sœurs de la Sainte-Famille de Sherbrooke se réfugièrent chez les Sœurs de la Présentation. Enfin, le 11 mars suivant, les étudiants reprenaicnt leurs cours dans les salles de l'ancien presbytère. Deux paroissiens avaient mis leurs demeures à la disposition du Collège pour les classes de rhétorique et de philosophie ${ }^{59}$. C'est ainsi que, tant bien que mal, les cours se poursuivirent jusqu'à la fin de l'année académique.

Après l'incendie de 1907, les gens de la ville de Saint-Jean vinrent trouver les prêtres du Petit Séminaire et leur demandèrent, après leur avoir fait miroiter certains avantages, de venir s'établir dans leur ville. M. Lemieux et les prêtres de Monnoir ne refusèrent pas l'offre qui venait de leur être faite. Cependant, il y avait une difficulté majeure à ce transfert : la ville de Saint-Jean était située dans le diocèse de Montréal, et non pas dans le diocèse de SaintHyacinthe, diocèsc auquel les prêtres de Monnoir appartenaient. L'évêque de Saint-Hyacinthe, $M^{\text {gr }}$ Bernard, s'opposa fermement à un tel transfert. Devant l'attitude de leur évêque, les prêtres de Monnoir décidèrent d'en appeler à Rome en disant que leurs droits étaient brimés.

Ce différend qui opposait l'évêque de Saint-Hyacinthe et les prêtres du Petit Séminaire de Sainte-Marie-de-Monnoir, au printemps de 1907 , va bientôt prendre des proportions considérables, surtout après que les prêtres eurent refusé de se soumettre à un premier jugement rendu par la Sacrée Congrégation de la Propagande. Devant cet état de fait, Rome demanda alors au Délégué apostolique, $\mathbf{M}^{\mathrm{gr}}$ Donat Sbaretti, de rendre un jugement final dans cette cause. Après avoir fait une enquête personnelle auprès des intéressés, $M^{\mathrm{gr}}$ Sbaretti rendit son jugement le 19 mars 1910. Mais c'était trop tard, les prêtres avaient déjà transféré le Collège Sainte-Marie-de-Monnoir dans la ville de Saint-Jean. L'affaire du Collège de Monnoir se retrouva lancée à nouveau.

Cette période de la vie du Collège Sainte-Marie-de-Monnoir demanderait à elle seule plus d'espace et de temps. Les événements

59 Ibid. 
qui se déroulèrent durant les années 1907-1912, demandant à être traités avec beaucoup de considération et d'analyse, nous ne pouvons dans le cadre du présent article aller plus avant dans les détails, nous le regrettons.

Le litige entre l'évêque de Saint-Hyacinthe et les prêtres de Monnoir se termina par la fermeture définitive du Collège et la dissolution de la Corporation. Parmi les 17 prêtres qui se trouvaient au Collège de Monnoir au moment de sa fermeture, seul l'abbé Bergeron demanda à rester dans le diocèse de Saint-Hyacinthe. Tous les autres demandèrent à $\mathrm{M}^{\mathrm{kr}}$ Bernard la permission de sortir $\mathrm{du}$ diocèse pour se chercher du ministère dans d'autres diocèses. M. Lemieux, pour sa part, se retira à Montréal.

La disparition du Collège de Monnoir du monde de l'enseignement fut bien regrettable, car cette institution offrait aux jeunes de la Province de Québec et d'ailleurs un programme d'études secondaires différent de celui des Collèges classiques traditionnels de l'époque, et répondait ainsi à un véritable besoin.

En terminant, nous tenons à remercier les responsables des archives de l'Évêché de Saint-Hyacinthe et du Petit Séminaire de Saint-Hyacinthe qui nous ont aidé et assisté dans cette recherche.

Jean-Jacques RoBILlaRD, O.P., Collège Dominicain de Philosophie et de Théologie, Ottawa, Ontario. 